\title{
COMPLEX INVERSION FORMULA FOR THE DISTRIBUTIONAL STIELTJES TRANSFORM
}

\author{
by STEVAN PILIPOVIĆ
}

(Received 19th September 1985)

\section{Introduction}

There are several approaches to the Stieltjes transform of generalized functions $([1$, $10,5,6,3,2])$. In this paper we use the definition of the distributional Stieltjes transform of index $\rho\left(\rho \in \mathbb{R} \backslash\left(-\mathbb{N}_{0}\right) ; \mathbb{N}_{0}=\mathbb{N} \cup\{0\}\right), S_{\rho}$-transform, given by Lavoine and Misra [3]. The $S_{\rho}$-transform is defined for a subspace of the Schwartz space $\mathscr{D}^{\prime}(\mathbb{R})$ while in $[10,5,6,2]$ the Stieltjes transform is defined for the elements of appropriate spaces of generalized functions. In these spaces differentiation is not defined which means that the Stieltjes transform of some important distributions, for example $\delta^{(k)}(x-a), a \geqq 0, k \in \mathbb{N}$, is meaningless in the sense of $[10,5,6,2]$. It is easy to see that the distributions $\delta^{(k)}(x-a)$, $a \geqq 0, k \in \mathbb{N}$, have the $S_{\rho}$-transform for $\rho>-k, \rho \in \mathbb{R} \backslash\left(-\mathbb{N}_{0}\right)$. These facts favour the approach to the Stieltjes transform given in [3].

The inversion theorems for the Stieltjes transform of generalized functions (in their own approaches) were given in $[1,10,6,7,2]$. We gave in [8] the so-called real inversion theorem for the $S_{\rho}$-transform.

The intention of this paper is to prove that the complex inversion theorem for the classical Stieltjes transform given by Sumner in [9] is also valid, with the convergence in $\mathscr{S}^{\prime}$ instead of pointwise convergence, for $S_{\rho} f, \rho>0$, where $f$ belongs to a subspace of the space of $S_{\rho}$-transformable tempered distributions. (As in [9] we suppose that $\rho>0$.)

\section{Definition of the $S_{\rho}$-transform}

Lavoine and Misra defined the Stieltjes transform by introducing the space $\mathscr{D}^{\prime}(a)$ in [3] and $\mathscr{J}^{\prime}(r)$ in [4]. As in [8] we give the definition of the $S_{\rho}$-transform in a little different notation.

The space $\mathscr{J}^{\prime}(\rho), \rho \in \mathbb{R} \backslash\left(-\mathbb{N}_{0}\right)$, is the space of distributions with supports in $[0, \infty)$ such that $f \in \mathscr{J}^{\prime}(\rho)$ iff there exists $k \in \mathbb{N}_{0}$ and locally integrable function $F$ with $\operatorname{supp} F \subset[0, \infty)$, such that

$$
\begin{gathered}
f=D^{k} F ; \\
\int_{0}^{\infty}|F(t)|(t+\beta)^{-\rho-k} d t<\infty \quad \text { for } \quad \beta>0
\end{gathered}
$$

( $D$ is the distributional derivative). Obviously, $\mathscr{J}^{\prime}(\rho) \subset \mathscr{J}^{\prime}(\rho+p), p \in \mathbb{N}_{0}$.

If instead of (2.1)(b) we suppose that there exist $C=C(F)$ and $\varepsilon=\varepsilon(F)>0$ such that

(c)

$$
|F(x)| \leqq C(1+x)^{\rho+k-1-\varepsilon} \quad \text { if } \quad x \geqq 0
$$


the corresponding space is denoted by $\mathscr{I}^{\prime}(\rho)$. Obviously $\mathscr{I}^{\prime}(\rho) \subset \mathscr{J}^{\prime}(\rho), \rho \in \mathbb{R} \backslash\left(-\mathbb{N}_{0}\right)$. The following implications hold ([8]):

$$
\begin{aligned}
& f \in \mathscr{J}^{\prime}(\rho), \rho+k>0 \Rightarrow f \in \mathscr{I}^{\prime}(\tilde{\rho}), \tilde{\rho}>\rho \text { for } \quad \tilde{\rho} \in \mathbb{R} \backslash\left(-\mathbb{N}_{0}\right) \\
& f \in \mathscr{J}^{\prime}(\rho), \rho+k<0 \Rightarrow f \in \mathscr{I}^{\prime}(\tilde{\rho}), \text { for } \rho>-k, \tilde{\rho} \in \mathbb{R} \backslash\left(-\mathbb{N}_{0}\right) .
\end{aligned}
$$

The Stieltjes transform $S_{\rho}, \rho \in \mathbb{R} \backslash\left(-\mathbb{N}_{0}\right)$, of a distribution $f \in \mathscr{J}^{\prime}(\rho)$ with properties given in $(2.1)(\mathrm{a}),(\mathrm{b})$, is a complex-valued function $\left(S_{\rho} f\right)$ defined by

$$
\left(S_{\rho} f\right)(s)=(\rho)_{k} \int_{0}^{\infty} F(t)(t+s)^{-\rho-k} d t, \quad s \in \mathbb{C} \backslash(-\infty, 0]
$$

where $(\rho)_{k}=\rho(\rho+1) \ldots(\rho+k-1), k>0$ and $(\rho)_{0}=1$.

For convenience we take that determination of $(s+t)^{-\rho-k}$ (also for any $z^{-\rho-s}, s \in \mathbb{N}_{0}$, which occurs in the paper) for which $\arg (s+t)^{-\rho-k}\left(\arg z^{-\rho-s}, s \in \mathbb{N}_{0}\right)$ has its principal value.

It is known ([4]) that $\left(S_{\rho} f\right)$ is a holomorphic function in $\mathbb{C} \backslash(-\infty, 0]$ and

$$
(\rho)_{p}\left(S_{\rho+p} f\right)(s)=\left(S_{\rho} f^{(p)}\right)(s), \quad f \in \mathscr{J}^{\prime}(\rho+p)
$$

( $f^{(p)}$ also denotes the $p$ th distributional derivative).

\section{Preliminary lemmas}

Let $\eta>0, t \in \mathbb{R}$. Following Sumner [9] we denote by $C_{\eta t}$, the contour in $\mathbb{C}$ which starts at the point $-t-i \eta$, proceeds along the straight line $\operatorname{Im} z=-\eta$ to the point $-i \eta$, then along the semicircle $|z|=\eta, \operatorname{Re} z \geqq 0$ to the point in and finally along the line $\operatorname{Im} z=\eta$ to the point $-t+i \eta$. We notice that these contours were observed in [9] only for $t>0$.

Let

$$
K(u, t)=K_{1}(t-u), \quad t, u \in \mathbb{R}, \quad t \neq u
$$

where

$$
K_{1}(x)=x^{-1}\left((\eta-i x)^{-\rho}-(\eta+i x)^{-\rho}\right), \quad x \in \mathbb{R} \backslash\{0\}, \quad \eta>0
$$

We need the following identities:

$$
\int_{C_{\eta t}} \frac{(z+t)^{\rho-1}}{(z+u)^{\rho+1}} d z=\frac{\eta^{\rho}}{\rho} K(u, t), u>0, \quad t \in \mathbb{R}, \quad t \neq u, \quad \eta>0 .
$$

(for $t>0$ this was quoted in $[9, \mathrm{p} .180]$ )

$$
\partial^{i} K(u, t) / \partial t^{i}=(-1)^{i} \partial^{i} K(u, t) / \partial u^{i}, \quad t, u \in \mathbb{R}, \quad u \neq t, \quad \eta>0
$$


By Leibniz formula we have

$$
\left|\partial^{i} K(u, t) / \partial t^{i}\right| \leqq C_{1} \sum_{r=0}^{i}|t-u|^{-i-1+r}\left(\eta^{2}+(t-u)^{2}\right)^{-(\rho+r) / 2}
$$

where $C_{1}=2 \max \left\{\left(\begin{array}{l}i \\ r\end{array}\right)(\mathrm{i}-\mathrm{r}) !(\rho)_{r} ; 0 \leqq r \leqq i\right\}$. Thus, with $C_{0}=(i+1) C_{1}$, there holds

$$
\left|\partial^{i} K(u, t) / \partial t^{i}\right| \leqq C_{0}|t-u|^{-i-1}\left(\eta^{2}+(t-u)^{2}\right)^{-\rho / 2}
$$

Up to the last section we shall suppose that $\rho>0$.

We denote by $\tilde{\mathscr{I}}^{\prime}(\rho)(\rho>0)$ a subset of $\tilde{I}^{\prime}(\rho+1)$ such that $f \in \tilde{I}^{\prime}(\rho)$ if in (2.1)(a) $F$ is continuous and if instead of $\left(2.1^{*}\right)(c)$ there holds

$$
|F(x)| \leqq C(1+x)^{\rho-\varepsilon}, \quad x \geqq 0 .
$$

for some $C>0$ and some $\varepsilon>0$. (We choose $\varepsilon$ such that $\rho-\varepsilon>0$.)

Lemma 1. Let $F$ be a continuous function on $\mathbb{R}$ with $\operatorname{supp} F \subset[0, \infty)$ and let $\left(2.1^{* *}\right)(\mathrm{d})$ hold. Then for every $k \in \mathbb{N}_{0}$ and $t_{0} \in \mathbb{R}$

$$
\left.\int_{-\infty}^{\infty} F(u)\left(\partial^{k} K(u, t) / \partial t^{k}\right)\right|_{t=t_{0}} d u=\left.\frac{d^{k}}{d t^{k}}\left(\int_{-\infty}^{\infty} F(u) K(u, t) d u\right)\right|_{t=t_{0}}
$$

Proof. Let $t_{0}>1$ and $I=\left[t_{0}-\frac{1}{2}, t_{0}+\frac{1}{2}\right]$. We shall prove that for any $i \in \mathbb{N}_{0}$, the function $\partial^{i} K(u, t) / \partial t^{i}$ is a continuous one for $(u, t) \in \mathbb{R} \times I, u \neq t$ (and has removable singularities on the set of measure zero in $\mathbb{R}^{2}$ ) and that the integral

$$
\int_{-\infty}^{\infty}\left|F(u) \partial^{i} K(u, t) / \partial t^{i}\right| d u
$$

converges uniformly on $I$. This will imply the assertion.

We split the last integral:

$$
J=J_{1}+J_{2}+J_{3}=\left(\int_{-\infty}^{t_{0}-1}+\int_{t_{0}-1}^{t_{0}+1}+\int_{t_{0}+1}^{\infty}\right)\left|F(u) \partial^{i} K(u, t) / \partial t^{i}\right| d u .
$$

From $\left(2.1^{* *}\right)(\mathrm{d})$ and (3.2) we obtain that the integrals $J_{1}$ and $J_{3}$ converge uniformly in $I$. Let

$$
H(u, t)= \begin{cases}K(u, t) & u \neq t, \quad(u, t) \in \mathbb{R}^{2} . \\ 2 \rho i / \eta^{\rho+1} & u=t,\end{cases}
$$

We shall prove that this function is a smooth one on $I_{1}=\left[t_{0}-1, t_{0}+1\right] \times\left[t_{0}-\frac{1}{2}, t_{0}+\frac{1}{2}\right]$. 
On setting $u=u, t=u+\eta \operatorname{tg} \phi$, we obtain

$$
\begin{aligned}
H(u, u+\eta \operatorname{tg} \phi) & =H_{1}(\phi)=\frac{2 i \cos ^{\rho+1} \phi \sin \rho \phi}{\eta^{\rho+1} \sin \phi} \\
& =\frac{2 i \cos ^{\rho+1} \phi}{\eta^{\rho+1}} \frac{\left(1-(\rho \phi)^{2} / 3 !+\cdots\right)}{\left(1-\phi^{2} / 3 !+\cdots\right)} .
\end{aligned}
$$

The function $H_{1}(\phi)$ is an analytic one in $[\operatorname{arctg}(-3 / 2 \eta)$, $\operatorname{arctg}(3 / 2 \eta)] \subset[-\pi / 2, \pi / 2]$. Since $\partial H / \partial t=\left(d H_{1} / d \phi\right)\left(\cos ^{2} \phi / \eta\right) \ldots$ we obtain that $H(u, t)$ is a smooth function in $I_{1}$.

If $0 \leqq t_{0} \leqq 1$, then we put

$$
J=\left(\int_{0}^{2}+\int_{2}^{\infty}\right)\left(\left|F(u) \partial^{i} K(u, t) / \partial t^{i}\right|\right) d u
$$

and by the same arguments as above one can prove that Lemma 1 holds.

If $t_{0}<0$, then the integrand in (3.3) is a smooth function of the variable $t$ on an interval $\left[t_{0}-h, t_{0}+h\right]$, where we choose $h$ such that $t_{0}+h<0$, and there is no longer the need to remove the singularity at $u=t$. So by $\left(2.1^{* *}\right)(\mathrm{d})$ and (3.2) the assertion of Lemma 1 follows.

Lemma 2. Let $F$ satisfy the conditions of Lemma 1, and let

$$
\phi_{i}(t)=\int_{-\infty}^{\infty}\left|F(u) \partial^{i} K(u, t) / \partial t^{i}\right| d u, \quad t \in \mathbb{R}, \quad i \in \mathbb{N}_{0}
$$

(i) There exist constants $K(i, \eta)$ and polynomials $p_{i}(t)$ such that

$$
\phi_{i}(t) \leqq K(i, \eta) p_{i}(t), \quad t \in \mathbb{R}, \quad i \in \mathbb{N}
$$

(Notice that $K(i, \eta)$ depends on $F$, as well.)

(ii) There exist a constant $K_{0}$ (which does not depend on $\eta$ ) and a polynomial $p_{0}(t)$ such that

$$
\eta^{\rho} \phi_{0}(t) \leqq K_{0} p_{0}(t), \quad t \in \mathbb{R}
$$

Proof. (i) Let $t>1$. We have

$$
\phi_{i}(t) \leqq\left(\int_{0}^{t-1}+\int_{t-1}^{t+1}+\int_{t+1}^{\infty}\right)\left(\left|F(u) \partial^{i} K(u, t) / \partial t^{i}\right|\right) d u=J_{1}+J_{2}+J_{3} .
$$


By (3.2) and $\left(2.1^{* *}\right)(d)$ we have

$$
\begin{aligned}
J_{1} & \leqq C C_{0} \int_{0}^{t-1} \frac{(1+u)^{\rho-\varepsilon}}{|t-u|^{i+1}\left(\eta^{2}+(t-u)^{2}\right)^{\rho / 2}} d u \\
& \leqq C C_{0}(1+t)^{\rho-\varepsilon} \int_{0}^{t-1} d u \leqq C C_{0}(1+t)^{\rho-\varepsilon} t
\end{aligned}
$$

because for $t>1$ and $u \in(0, t-1),(t-u)^{i+1}\left(\eta^{2}+(t-u)^{2}\right)^{\rho / 2}>1$.

$$
\begin{aligned}
J_{3} & \leqq C C_{0} \int_{t+1}^{\infty} \frac{(1+u)^{\rho-\varepsilon} d u}{|t-u|^{i+1}\left(\eta^{2}+(t-u)^{2}\right)^{\rho / 2}} \\
& =C_{0} C \int_{0}^{\infty} \frac{(2+t+v)^{\rho-\varepsilon} d v}{(v+1)^{i+1}\left(\eta^{2}+(v+1)^{2}\right)^{\rho / 2}}
\end{aligned}
$$

Since $\rho-\varepsilon>0$, from $(2+t+v)^{\rho-\varepsilon} \leqq 2^{\rho-\varepsilon}\left((2+t)^{\rho-\varepsilon}+v^{\rho-\varepsilon}\right), v>0$, we obtain

$$
J_{3} \leqq 2^{\rho-\varepsilon} C_{0} C\left((2+t)^{\rho-\varepsilon} \int_{0}^{\infty} \frac{d v}{(v+1)^{i+1}\left(\eta^{2}+(v+1)^{2}\right)^{\rho / 2}}+\int_{0}^{\infty} \frac{v^{\rho-\varepsilon} d v}{(v+1)^{i+1}\left(\eta^{2}+(v+1)^{2}\right)^{\rho / 2}}\right)
$$

For $J_{2}$ we have

$$
\begin{aligned}
J_{2} \leqq & \sup _{t-1 \leqq u \leqq t+1}\{|F(u)|\} \int_{t-1}^{t+1}\left|\partial^{i} K(u, t) / \partial t^{i}\right| d u \\
& \leqq C(t+2)^{\rho-\varepsilon} \int_{-1}^{1}\left|\partial^{i} K(0, s) / \partial s^{i}\right| d s .
\end{aligned}
$$

Since the function $H(0, s), s \in[-1,1]$, where $H(u, t)$ is defined in the proof of Lemma 1 , is a smooth one, we obtain that for some constant $M_{i}$ which depends on $\eta$

$$
J_{2} \leqq M_{i}(t+2)^{\rho-\varepsilon}
$$

Estimations for $J_{1}, J_{2}$ and $J_{3}$ imply that the assertion holds if $t>1$.

Let $0 \leqq t \leqq 1$. Then we have

$$
\phi_{i}(t) \leqq\left(\int_{0}^{2}+\int_{2}^{\infty}\right)\left(\left|F(u) \partial^{i} K(u, t) / \partial t^{i}\right|\right) d u
$$

and by the similar arguments as above one can prove that the assertion holds.

If $t<0$, there is no need to split the integral in (3.4) and the assertion follows by the arguments given above. 
(ii) Let $t>1$. From the first part of this lemma we conclude that only in the estimation of the integral $J_{2}$, the constant $K(i, \eta)$ really depends on $\eta$. But, on setting $s=\eta \operatorname{tg} \phi$ in

$$
\int_{-1}^{1}\left|\partial^{i} K(0, s) / \partial s^{i}\right| d s
$$

in the same way as in the proof of Lemma 4(b) from [9], one can prove the assertion. For $t \leqq 1$ we have to use arguments given above.

Lemma 3. Let $F$ be a continuous function on $\mathbb{R}$ with $\operatorname{supp} F \subset[0, \infty)$ and let $\left(2.1^{* *}\right)(\mathrm{d})$ hold. Then

$$
\lim _{\eta \rightarrow 0^{+}} \frac{\eta^{\rho}}{2 i \pi} \int_{-\infty}^{\infty} F(u) K(u, t) d u=F(t), \quad t \in \mathbb{R}
$$

Proof. For $t \geqq 0$ the proof follows from [9, Lemma 4(c)] since for enough large $R$

$$
\lim _{\eta \rightarrow 0^{+}} \frac{\eta^{\rho}}{2 i \pi} \int_{R}^{\infty} F(u) K(u, t) d u=0
$$

Since $\rho>0$ and

$$
\int_{0}^{\infty}|F(u) K(u, t)| d u \leqq \int_{0}^{\infty}|F(u)|(|t|+u)^{-\rho-1} d u<\infty
$$

we obtain

$$
\lim _{\eta \rightarrow 0^{+}} \frac{\eta^{\rho}}{2 i \pi} \int_{-\infty}^{\infty} F(u) K(u, t) d u=0, \quad t<0
$$

The proof is complete.

If $f \in \mathscr{I}^{\prime}(\rho)$, then for $t \in \mathbb{R}$ we have

$$
\begin{aligned}
\rho \int_{C_{\eta t}}(z+t)^{\rho-1}\left(S_{\rho+1} f\right)(z) d z \\
\quad=(\rho)_{k+1} \int_{C_{\eta t}}(z+t)^{\rho-1}\left(S_{\rho+k+1} F\right)(z) d z \\
\quad=(\rho)_{k+1} \int_{C_{\eta t}}(z+t)^{\rho-1}\left(\int_{-\infty}^{\infty} \frac{F(u) d u}{(z+u)^{\rho+k+1}}\right) \\
=(\rho)_{k+1} \int_{-\infty}^{\infty} F(u)\left(\int_{C_{\eta t}} \frac{(z+t)^{\rho-1} d z}{(z+u)^{\rho+k+1}}\right) d u
\end{aligned}
$$


The last equality holds on the basis of the uniform convergence of

$$
\int_{-\infty}^{\infty} \frac{F(u)}{(z+u)^{\rho+k+1}} d u \text { for } z \in C_{\eta t}
$$

Thus we have (by (3.1))

$$
\rho \int_{C_{\eta \prime}}(z+t)^{\rho-1}\left(S_{\rho+1} f\right)(z) d z=(-1)^{k} \eta^{\rho} \int_{-\infty}^{\infty} F(u)\left(\partial^{k} K(u, t) / \partial u^{k}\right) d u, \quad t \in \mathbb{R} .
$$

Lemma 2 implies that this integral defines a tempered distribution.

\section{The main result}

Theorem 4. Let $f \in \tilde{I}^{\prime}(\rho)$. Then for every $\phi \in \mathscr{S}$

$$
\lim _{\eta \rightarrow 0^{+}}\left(\frac{\rho}{2 i \pi}\left\langle\int_{c_{\eta t}}(z+t)^{\rho-1}\left(S_{\rho+1} f\right)(z) d z, \phi(t)\right\rangle\right)=\langle f(t), \phi(t)\rangle
$$

Proof. We have

$$
\begin{aligned}
\frac{\rho}{2 i \pi}\left\langle\int_{C_{\eta t}}(z+t)^{\rho-1}\left(S_{\rho+1} f\right)(z) d z, \phi(t)\right\rangle & \quad \text { (by (2.3)) } \\
& =\frac{1}{2 i \pi}(\rho)_{k+1}\left\langle\int_{C_{\eta t}}(z+t)^{\rho-1}\left(S_{\rho+k+1} F\right)(z) d z, \phi(t)\right\rangle \\
& =\frac{(\rho)_{k+1}}{2 i \pi}\left\langle\int_{C_{n t}}(z+t)^{\rho-1}\left(\int_{-\infty}^{\infty} \frac{F(u)}{(z+u)^{\rho+k+1}} d u\right) d z, \phi(t)\right\rangle \quad \text { (by the uniform convergence) } \\
& =\frac{(\rho)_{k+1}}{2 i \pi}\left\langle\int _ { - \infty } ^ { \infty } F ( u ) \left(\int_{C_{\eta t}} \frac{(z+t)^{\rho-1}}{\left.\left.(z+u)^{\rho+k+1} d z\right) d u, \phi(t)\right\rangle} \quad\right.\right. \text { (by the uniform convergence) } \\
& =\frac{(-1)^{k} \rho}{2 i \pi}\left\langle\int_{-\infty}^{\infty} F(u)\left(\frac{\partial^{k}}{\partial u^{k}} \int_{C_{\eta t}} \frac{(z+t)^{\rho-1}}{(z+u)^{\rho+1}} d z\right) d u, \phi(t)\right\rangle, \quad \\
& =(-1)^{k} \frac{\eta^{\rho}}{2 i \pi}\left\langle\int_{-\infty}^{\infty} F(u)\left(\partial^{k} K(u, t) / \partial u^{k}\right) d u, \phi(t)\right\rangle \\
= & \frac{\eta^{\rho}}{2 i \pi}\left\langle\int_{-\infty}^{\infty} F(u)\left(\partial^{k} K(u, t) / \partial t^{k}\right) d u, \phi(t)\right\rangle,
\end{aligned}
$$$$
=\frac{(-1)^{k} \rho}{2 i \pi}\left\langle\int_{-\infty}^{\infty} F(u)\left(\frac{\partial^{k}}{\partial u^{k}} \int_{c_{n t}} \frac{(z+t)^{\rho-1}}{(z+u)^{\rho+1}} d z\right) d u, \phi(t)\right\rangle, \quad \text { (by the uniform convergence) }
$$ 


$$
\begin{aligned}
& =\frac{\eta^{\rho}}{2 i \pi} \int_{-\infty}^{\infty}\left(\int_{-\infty}^{\infty} F(u)\left(\partial^{k} K(u, t) / \partial t^{k}\right) d u\right) \phi(t) d t \\
& =\frac{\eta^{\rho}}{2 i \pi} \int_{-\infty}^{\infty} \frac{d^{k}}{d t^{k}}\left(\int_{-\infty}^{\infty} F(u) K(u, t) d u\right) \phi(t) d t \\
& =\frac{\eta^{\rho}(-1)^{k}}{2 i \pi} \int_{-\infty}^{\infty}\left(\int_{-\infty}^{\infty} F(u) K(u, t) d u\right) \phi^{(k)}(t) d t
\end{aligned}
$$

(by partial integration).

Thus, by Lemma 3, Lemma 2(ii) and Lebesque's theorem we obtain

$$
\lim _{\eta \rightarrow 0^{+}} \frac{\rho}{2 i \pi}\left\langle\int_{C_{\eta t}}(z+t)^{\rho-1}\left(S_{\rho+1} f\right)(z) d z, \phi(t)\right\rangle=(-1)^{k}\left\langle F(t), \phi^{(k)}(t)\right\rangle=\langle f, \phi\rangle .
$$

The proof is complete.

Let us notice that if $f \in \tilde{I}^{\prime}(\rho) \cap \mathscr{I}^{\prime}(\rho)$ then we have $-\rho\left(S_{\rho+1} f\right)=\left(S_{\rho} f\right)^{\prime}$, and Theorem 4 can be formulated in a way more similar to [9].

Example. Let $\rho \in \mathbb{P} \backslash\left(-\mathbb{N}_{0}\right), \rho>1$ and $a \geqq 0$.

$$
\delta^{(k)}(x-a) \in \tilde{I}^{\prime}(\rho)
$$

since

$$
\delta^{(k)}(x-a)=F^{(k+2)}(x) \text { where } F= \begin{cases}x-a, & x \geqq a \\ 0, & x<a\end{cases}
$$

We have

$$
S_{\rho}\left(\delta^{(k)}(x-a)\right)(s)=(\rho)_{k} \frac{1}{(a+s)^{\rho+k}}, \quad s \in \mathbb{C} \backslash(-\infty, 0]
$$

Theorem 4 implies that for the distribution determined by the function

$$
\begin{gathered}
\phi_{\eta}(t)=\frac{1}{2 i \pi} \int_{C_{\eta t}}(z+t)^{\rho-1}(\rho)_{k+1} \frac{1}{(a+z)^{\rho+k+1}} d z, \quad t \in \mathbb{R}, \quad \eta>0, \\
\lim _{\eta \rightarrow 0^{+}} \phi_{\eta}(t)=\delta^{(k)}(x-a) \text { in } \mathscr{S}^{\prime} .
\end{gathered}
$$




\section{Remarks}

There is an easy way to give the complex inversion formula for $S_{\rho} f$, where $\rho \in \mathbb{R} \backslash\left(-\mathbb{N}_{0}\right)$ and $f \in \mathscr{I}^{\prime}(\rho)$. This formula is not so "natural" as the quoted one in Theorem 4 but we shall give it here for the sake of completeness.

If $f \in \mathscr{I}^{\prime}(\rho)\left((2.1)(\mathrm{a})\right.$ and $\left(2.1^{*}\right)(\mathrm{c})$ hold), $\rho \in \mathbb{P} \backslash\left(-\mathbb{N}_{0}\right)$ and if $F$ is a continuous function then, using [9, Theorem $4(\mathrm{a})]$ and Lemmas 2 and 3, one can easily prove that

$$
\begin{aligned}
& -\frac{1}{2 i \pi(\rho)_{k+1}}\left\langle\frac{d^{k}}{d t^{k}}\left(\int_{C_{n t}}(z+t)^{\rho+k-1}\left(S_{\rho} f\right)^{\prime}(z) d z\right), \phi(t)\right\rangle \\
& =-(-1)^{k} \frac{1}{2 i \pi}\left\langle\int_{C_{\eta t}}(z+t)^{\rho+k-1}\left(S_{\rho+k+1} F\right)^{\prime}(z) d z, \phi^{(k)}(t)\right\rangle
\end{aligned}
$$

converges to

$$
(-1)^{k}\left\langle F(t), \phi^{(k)}(t)\right\rangle=\langle f(t), \phi(t)\rangle
$$

as $\eta \rightarrow 0^{+}(\phi \in \mathscr{S})$.

Acknowledgement. The author would like to thank the referee for many helpful comments. This material is based on work supported by the U.S.-Yugoslav Joint Fund for Scientific and Technological Co-operation, in co-operation with NSF under grant JFP 544.

\section{REFERENCES} 319.

1. J. J. Benedetro, Analytic representation of generalised functions, Math. Z. 97 (1967), 303-

2. A. ERdÉlyı, Stieltjes transform of generalized functions, Proc. Roy. Soc. Edinburgh 76A (1977), 231-249.

3. J. Lavoine and O. P. Misra, Théorèmes abéliens pour la transformation de Stieltjes des distributions, C.R. Acad. Sci. Paris, Série A 279 (1974), 99-102.

4. J. Lavoine and O. P. MisRa, Abelian theorems for the distributional Stieltjes transformation, Math. Proc. Cambridge Philos. Soc. 86 (1979), 287-293.

5. O. P. Misra, Some Abelian theorems for distributional Stieltjes transformation, J. Math. Anal. Appl. 39 (1972), 590-599.

6. J. N. Pandey, On the Stieltjes transform of generalized functions, Math. Proc. Cambridge Philos. Soc. 71 (1971), 85-96.

7. R. S. Pathak, A distributional generalized Stieltjes transformation, Proc. Edinburgh Math. Soc. 70 (1976), 15-22.

8. S. PILIPovic, An inversion theorem for the distributional Stieltjes transformation of distributions, Proc. Edinburgh Math. Soc., 29 (1986), 171-185.

9. D. B. SUMner, An inversion formula for the generalized Stieltjes transform, Bull. Amer. Math. Soc. 55 (1949), 174-183.

10. A. H. Zemanian, Generalized Integral Transformations (Interscience, New York, 1968).

InSTITUTE OF MATHEMATICS

UNIVERSITY OF NOVI SAD

YUGOSLAVIA 